\title{
Acute exposure to paraquat affects the phenotypic differentiation of substantia nigra microglia in rats
}

\author{
Wendi Zhang \\ Hebei Medical University \\ Xiaobei Fan \\ Hebei Medical University \\ Zhuo Fan \\ Hebei Medical University

\section{Bailin Wu} \\ Hebei Medical University No2 Hospital: Second Hospital of Hebei Medical University

\section{Mengchao Wang \\ Hebei Medical University \\ Wanyu Duan \\ Hebei Medical University} \\ Bo Song ( $\square$ songbootc@163.com ) \\ Hebei Medical University https://orcid.org/0000-0001-5933-7511
}

\section{Research Article}

Keywords: paraquat, microglia, phenotypic differentiation, acute exposure

Posted Date: February 24th, 2021

DOI: https://doi.org/10.21203/rs.3.rs-201966/v1

License: (c) (1) This work is licensed under a Creative Commons Attribution 4.0 International License.

Read Full License

Version of Record: A version of this preprint was published at Environmental Science and Pollution Research on November 10th, 2021. See the published version at https://doi.org/10.1007/s11356-02117262-3. 


\section{Abstract}

The toxicity of paraquat (PQ), a bipyridine cationic herbicide, to the lung and kidney has been widely recognized, but the acute toxic effects of $P Q$ on the nervous system have received little attention. The aim of this study was to explore changes in the phenotypic differentiation of microglia in rats caused by acute $P Q$ exposure. We found that acute $P Q$ exposure induced pyknosis, edema and apoptosis in substantia nigra neurons. The results of immunohistochemistry and western blotting showed that the number of activated microglia increased, the number of microglia branches decreased and the length became shorter in the early stage of exposure to $25 \mathrm{mg} / \mathrm{kg} \mathrm{PQ}$. We then compared the mean fluorescence intensity of iNOS and ARG1. In the early stage of acute $25 \mathrm{mg} / \mathrm{kg} P Q$ exposure, the number of M1 phenotypes of microglia increased; in contrast, in the early stage of acute $45 \mathrm{mg} / \mathrm{kg} \mathrm{PQ}$ exposure, the number of M2 phenotypes of microglia increased. On the 69th day, the expression of iNOS and ARG1 increased in the $25 \mathrm{mg} / \mathrm{kg}$ and $45 \mathrm{mg} / \mathrm{kg}$ PQ exposure groups. In sum, changes in microglia phenotypic differentiation were related to exposure dose and exposure time.

\section{Introduction}

Paraquat (PQ) is a bipyridine cationic broad-spectrum herbicide that is a common cause of acute poisoning, with a mortality rate greater than $50 \%$ (Sun et al, 2018). Numerous studies have shown that PQ can enter the brain through the blood-brain barrier and exist in ionic form (Bonneh-Barkay et al 2005, Shimizu et al 2001). Long-term exposure to PQ can lead to Parkinson-like symptoms (Brooks et al 1999, McCormack et al 2002). PQ is thought to induce the oxidative stress response and inhibit the activity of mitochondrial complex I, inducing neuron injury and apoptosis in the striatum and substantia nigra (SN) (Cassar et al 2015, Rodriguez-Rocha et al 2013, Zhang et al 2016). We previously have explored the acute neurotoxicity of $\mathrm{PQ}$ and its mechanism using hydrogen proton magnetic resonance spectroscopy $(1 \mathrm{H}$ MRS) and magnetic resonance diffusion tensor imaging (DTI) techniques. We observed the brain morphology and function of 26 patients with acute $P Q$ poisoning and found that after 3-7 days of acute $P Q$ poisoning, mild edema occurred in some brain regions, such as the frontal, parietal, basal ganglia and hippocampus. The myelin sheath in the substantia nigra pars reticularis, substantia nigra pars compacta, red nucleus and hippocampus showed certain structural abnormalities, the cerebral blood flow in the midbrain and frontal lobe decreased and the ratio of the brain metabolites inositol (MI), lipids (Lip) and phosphocreatine $(\mathrm{Cr})$ in the basal ganglia increased (Wu et al 2012). Our previous studies have confirmed that acute $P Q$ exposure leads to structural and functional abnormalities in the central nervous system.

To explore its possible mechanism, we established a rat acute $P Q$ poisoning model. The preliminary results showed that neurons in the different brain regions of rats showed oxidative stress damage and apoptosis effects in the early stage of poisoning, and the expression of oxidative stress products, such as 8-hydroxydeoxyguanosine (8-OHdG), superoxide dismutase (SOD) and malondialdehyde (MDA), was increased. Abnormal morphology, cytoplasmic edema and apoptosis of neurons were observed in the cerebral cortex, striatum and hippocampus by a transmission electron microscope (Wu et al 2013, Wu \& Song 2013a, Wu \& Song 2013b). Recent studies have also shown that microglia mediate the selective 
damage of $P Q$ to $D A$ neurons after low-dose $P Q$ exposure in the co-culture of microglia and neurons. Specifically, low-dose PQ exposure induces the production of NADPH oxidase and peroxides in microglia, thus resulting in selective damage to DA neurons (Kim et al 2008, Wu et al 2005). Other studies have also shown that neuronal damage is closely related to microglia (Gomes-Leal 2019, Rodriguez-Iglesias et al 2019 , Sosa et al 2019). We thus hypothesized that microglia may play a key role in affecting neuronal damage and repair in the acute neurotoxicity of $P Q$.

In this study, we observed microglia labeled with Iba-1 and analyzed the average endpoints and process length of Iba-1 positive cells in SN after exposure to $P Q$ in female rats. In addition, changes in the expression of iNOS and ARG1 were used to reflect the different activation degrees of M1 and M2 phenotypes. Details on the mechanism of the acute neurotoxicity of $P Q$ were elucidated by observing changes in microglia after acute PQ exposure.

\section{Materials And Methods}

\subsection{Experimental animal and model establishment}

A total of 36 adult female Wistar rats ( $180 \mathrm{~g}$ to $200 \mathrm{~g}$ in weight) were provided by the Animal Experimental Center of Hebei Medical University (certificate number:1810100). Animals were acclimated to the environment for one week before experiments. The animals were randomly assigned to the control group (normal saline group, $\mathrm{n}=12), 25 \mathrm{mg} / \mathrm{kg} P Q$ exposure group $(\mathrm{n}=12)$ and $45 \mathrm{mg} / \mathrm{kg} P Q$ exposure group $(n=12)$. A single exposure was performed on each group of experimental animals through oral administration. Clean water and standard food were provided throughout the entire experiment. The food was purchased from the Animal Experimental Center of Hebei Medical University. The management and disposal of laboratory animals in this study were consistent with the National Institutes of Health guidelines for the care and use of laboratory animals (National Institutes of Health Publication No. 8023, revised 1978). Animals were observed for changes in general behavior and body weight after exposure, and materials were drawn on the 18th, 39th and 69th days of exposure.

\subsection{Brain tissue sampling and fixation}

After the rats were anesthetized with $3 \%$ pentobarbital, the thoracic cavity was opened to expose the heart. The needle was inserted from the apex into the aorta through the left ventricle, the right atrial appendage was cut and $150 \mathrm{ml}$ of normal saline at $4{ }^{\circ} \mathrm{C}$ was quickly injected until the liver became white and the effluent liquid was clear. Next, $250 \mathrm{ml}$ of frozen $4 \%$ paraformaldehyde was perfused to rats gradually. After the end of the perfusion, the brain tissue was removed and placed into $4 \%$ paraformaldehyde for post-fixation. After fixation, the tissue was subjected to gradient dehydration by $70 \%, 80 \%, 90 \%$ and $100 \%$ ethanol, made transparent by xylene and then was embedded in paraffin.

\subsection{Pathological changes in brain tissue}

Brain tissue 5 um in thickness was dried and baked in a drying oven at $60^{\circ} \mathrm{C}$ for 2 hours. After dewaxing with xylene and gradual alcohol hydration, it was stained with hematoxylin for 10 minutes and then 
rinsed with running water. The sections were differentiated and turned blue after the above treatment, followed by staining with eosin for 5 minutes and rinsing. Next, the sections were dehydrated by gradient alcohol, made transparent with xylene and then were sealed with a neutral gum. The pathological changes of the hippocampus, striatum and SN in brain tissue were then observed.

\subsection{Immunohistochemical (IHC) staining}

After the sections were hydrated, they were placed into $0.01 \mathrm{~mol} / \mathrm{L}$ citrate buffer and heated in a water bath at $95^{\circ} \mathrm{C}$ for 30 minutes to repair the antigen. The sections were then blocked with $3 \%$ hydrogen peroxide for 10 minutes and $5 \%$ goat serum for 40 minutes until they cooled to room temperature (RT) and were rinsed with PBS. The sections were then incubated with rabbit anti-lba-1 (1:1000, Wako Pure Chemical, Japan) at $4^{\circ} \mathrm{C}$ overnight and with biotinylated goat anti-rabbit IgG for 1 hour at RT after washing. Next, the sections were incubated with avidin-biotin peroxidase complex (1:200, ABC elite kit, Vector Laboratories, USA) for 30 minutes at RT and 0.5\% 3-3-diaminobenzidine tetrachloride (DAB, Vector Laboratories, USA) for 3-10 min. The reaction was stopped by extensive washing in PBS, and the nuclei were counterstained with hematoxylin. PBS was replaced with PBS as a negative control.

\subsection{Immunofluorescence (IF) double staining}

After the sections were subjected to antigen retrieval, they were washed with PBS and then were blocked with $5 \%$ horse serum for 40 minutes. After washing with PBS, sections were incubated overnight at $4{ }^{\circ} \mathrm{C}$ with rabbit anti-lba-1 (1:1000) and mouse anti-ARG1 (1:50, Santa Cruz, USA) or anti-iNOS (1:50,Santa Cruz, USA); slices were washed in PBS and incubated with Donkey Anti-Mouse IgG H\&L (Alexa Fluor ${ }^{\circledR}$ 568, 1:800; Abcam, UK) and Donkey Anti-Rabbit IgG H\&L (Alexa Fluor ${ }^{\circledR} 488,1: 800$; Abcam, UK) for $1 \mathrm{~h}$ at RT in the dark. After rinsing with PBS several times, VECTASHIELD® Mounting Medium with DAPI (VECTOR, USA) was added to the slices for staining and sealing.

\subsection{Western blot}

The SN of brain was isolated and homogenized in lysis buffer (Multi Science, China). After the centrifugation of the homogenates at 14,000 rpm for $5 \mathrm{~min}$, the supernatants were harvested. Proteins were separated by sodium dodecyl sulfate-polyacrylamide gel electrophoresis (SDS-PAGE), transferred to a polyvinylidene fluoride membrane (Bio-Rad Laboratories Inc., Hercules, CA), probed with the indicated antibodies and detected with enhanced chemiluminescence.

\subsection{Data analysis}

The results of $\mathrm{HE}$ and IHC staining in SN $(4.80 \mathrm{~mm}$ behind the bregma and $4.20 \mathrm{~mm}$ in front of the interaural line) were observed using an OLYMPUS BX-53 microscope (Olympus Corporation, Japan); 3 to 5 fields of each slice were obtained at low magnification $(10 \times 10)$ and high magnification $(20 \times 10)$. Images of IF double labeling were taken using OLYMPUS BX-53 and U-RFL-T microscopes (Olympus Corporation, Japan). The number of Iba-1 positive cells was counted, and the morphology of microglia was analyzed using Fiji image J 2.0 (Healy et al 2018, Hirayama et al 2018, Schindelin et al 2012, Young \& Morrison 2018). The mean fluorescence intensity (MFI) of IF staining was calculated. Data were 
expressed as mean \pm SD, and statistical analyses were performed with SPSS version 26.0. Differences among groups were evaluated by one-way ANOVA when appropriate. Least significant difference tests were also used to make multiple comparisons. $P<0.05$ was the threshold for statistical significance. GraphPad Prism 7.0 software was used to plot graphs.

\section{Results}

\subsection{Changes in the general conditions of animals after acute $\mathrm{PQ}$ exposure}

After 1 to 2 days of exposure, both the 25 and $45 \mathrm{mg} / \mathrm{kg} \mathrm{PQ}$ exposure groups showed symptoms of mouth corner and canthus hemorrhage. Some rats showed shortness of breath with murmurs, poor mental state and reduced activity. After acute PQ exposure, the weight of rats in the 25 and $45 \mathrm{mg} / \mathrm{kg} \mathrm{PQ}$ exposure groups decreased and gradually recovered after 5 days (Fig. 1.). Differences between these groups were not statistically significant $(P>0.05)$.

\subsection{Histopathological changes in SN after acute PQ exposure}

The results of HE staining (Fig. 2.) showed that the neurons in $\mathrm{SN}$ were loosely arranged after acute $\mathrm{PQ}$ exposure. Nuclear pyknosis, edema and neuronophagocytosis were observed, especially in the $45 \mathrm{mg} / \mathrm{kg}$ $P Q$ exposure group.

\subsection{Morphology of Iba-1 positive cells}

The results of IHC staining labeled with Iba-1 (Fig. 3.) showed that microglia had small cell bodies and long branches in the resting state. After activation, the cell bodies became larger, and the branches became shorter or even disappeared, exhibiting "amoeba-like" changes.

\subsection{The number of Iba-1 positive cells}

The number of Iba-1 positive cells was counted under high magnification (200x) given the role of markers of Iba-1 for microglia (Kadowaki et al 2007). The number of Iba-1 positive cells in the SN was significantly increased in the $45 \mathrm{mg} / \mathrm{kg} \mathrm{PQ}$ group on the 39th and 69th day of exposure compared with the control group $(\mathrm{P}<0.05)$ (Fig. 4. A-C). Furthermore, the number of endpoints was significantly decreased in the $25 \mathrm{mg} / \mathrm{kg} P Q$ group on the 39th day but was significantly increased on the 69th day of exposure compared with the control group $(P<0.05)$. The process length of Iba- 1 positive cells was significantly decreased in the $25 \mathrm{mg} / \mathrm{kg} \mathrm{PQ}$ group on the 18th and 39th day but was significantly increased on the 69th day of exposure relative to the control group $(P<0.05)$. The process length was significantly decreased in the $45 \mathrm{mg} / \mathrm{kg} P Q$ group on the 39th day of exposure relative to the control group $(P<0.05)$. Western blotting was then used to detect the expression of Iba- 1 (Fig. 4D-F). Iba-1 expression was significantly decreased in the $25 \mathrm{mg} / \mathrm{kg} \mathrm{PQ}$ group on the 39th day of exposure but was significantly increased in the $45 \mathrm{mg} / \mathrm{kg} P Q$ group on the 39th day of exposure $(P<0.05)$. 


\subsection{Changes in microglia activation balance after acute PQ exposure}

The results of IF staining labeled with iNOS (Fig. 5A-B) showed that the expression of iNOS in the 45 $\mathrm{mg} / \mathrm{kg} P Q$ group was significantly increased on the 69th day of exposure in the $\mathrm{SN}$ compared with the control group $(P<0.05)$. The expression of ARG1 was significantly increased in the $45 \mathrm{mg} / \mathrm{kg}$ group on the 18th and 69th day of exposure compared with the control group $(P<0.05)$. The expression of ARG1 in the $25 \mathrm{mg} / \mathrm{kg}$ PQ group was significantly increased only on the 39th day of exposure compared with the control group $(P<0.05)$. Western blotting was used to detect the expression of iNOS and ARG1 (Fig. 5CE). The expression of iNOS was significantly increased in the $25 \mathrm{mg} / \mathrm{kg} \mathrm{PQ}$ group on the 18th and 69th day of exposure and in the $45 \mathrm{mg} / \mathrm{kg} P Q$ group on the 69th day of exposure in the SN compared with the control group $(P<0.05)$ (Fig. 5. C-E). The expression of ARG1 was significantly increased in the $45 \mathrm{mg} / \mathrm{kg}$ $P Q$ group on the 18th and 69th day of exposure and in the $25 \mathrm{mg} / \mathrm{kg} \mathrm{PQ}$ group on the 39th day of exposure compared with the control group $(P<0.05)$.

\section{Discussion}

In this study, we demonstrated that acute exposure to $P Q$ could affect the phenotypic differentiation of SN microglia in female rats. We observed the expression of Iba-1, iNOS and ARG1 in the SN in female rats at different $P Q$ doses and inferred the relationship between the change in the microglia polarization phenotype and the dose of acute PQ exposure at different times.

Some studies have shown sex specificity in the number, morphology and distribution of microglia in adult mice under various physiological conditions, but significant sexual differences in the expression of iNOS and ARG1 have yet to be reported (Crain et al 2013, Lawson et al 1990, Mouton et al 2002, Villa et al 2019). In this study, the role of microglia in acute $P Q$ central neurotoxicity was studied in female rats.

In the early stage of acute $\mathrm{PQ}$ exposure, the $\mathrm{SN}$ had degenerated neurons showing cell body edema and nuclear pyretic contraction, and the neurons were loosely arranged because of nerve phagocytosis; this morphology was most evident in the $45 \mathrm{mg} / \mathrm{kg} P Q$ group. This damage to neurons has been suggested to be related to the activation of microglia. Additional experiments have shown that these neuronal injuries are related to the oxidative stress of microglia (Barlow et al 2003, Miller et al 2007, Wu et al 2005). Neurons can bind to the corresponding receptors of microglia by releasing soluble immunomodulatory factors (e.g., CX3CL1, TGF- $\beta$, glutamate, and NE) to induce changes in microglia function and morphology. Meanwhile, microglia can affect neuronal function by releasing cytokines, such as IL-1 $\beta$ and TNF-a, (Sheridan \& Murphy 2013, Wohleb 2016). Therefore, microglia are an important factor in the neurotoxicity of neurons and are the key cells associated with the oxidative stress induced by PQ (Wu et al 2005, Chen et al 2015).

Numerous studies have shown that microglia operating as immune cells inherent in the brain could be activated rapidly after nervous system homeostasis is destroyed and thus alter their phenotype and 
function. The ionized calcium-binding adaptor molecule 1 (lba-1) is a specific marker for microglia (Kawasaki et al 2007). In this study, microglia cells were labeled with lba-1 to observe morphological changes after exposure to PQ. To quantify changes in the morphology of microglia, ImageJ was used for the binary conversion of microglia images, and the cytoskeleton was drawn and analyzed. Two important indexes, the average number of microglia branches and the average branch length, were used for analysis (Young et al 2018, Schindelin et al 2012). We found that after acute PQ exposure, the number of activated microglia in SN was increased on the 39th and 69th days after exposure. The process length of microglia decreased in the $25 \mathrm{mg} / \mathrm{kg} \mathrm{PQ}$ group on the 18th day. Therefore, we speculate that microglia activation is induced by low-dose $P Q$ in the early stage of $P Q$ exposure. Microglia were still activated after a long period of time, suggesting that the neurotoxicity of $P Q$ can persist for long periods.

Having determined that microglia were activated after acute PQ exposure, we next sought to examine the phenotypic differentiation of SN microglia. Microglia are classified based on their metabolites, namely M1 and M2 phenotypes (Jha et al 2016, Mantovani et al 2004, Nakagawa \& Chiba 2014). M1 phenotype microglia are characterized by the production of pro-inflammatory factors leading to cell damage. Inducible nitric oxide synthase (iNOS) is a specific product of the M1 phenotype, which can thus be used as a marker of the M1 phenotype (Cherry et al 2014, Chhor et al 2013). The M2 phenotype mainly secretes anti-inflammatory factors and neurotrophic factors, which can repair or protect neurons. Arginase enzyme 1 (ARG1) is the most specific marker of the M2 phenotype (Cherry et al 2014). On the 18th day after exposure to $P Q$, the expression of iNOS increased in the $25 \mathrm{mg} / \mathrm{kg} \mathrm{PQ}$ group, and the expression of ARG1 increased significantly in the $45 \mathrm{mg} / \mathrm{kg} P Q$ group. Thus, in the early stage of acute $P Q$ exposure, low-dose $P Q$ exposure can increase the number of $M 1$ phenotypes of microglia, whereas high-dose $P Q$ exposure can increase the number of $M 2$ phenotypes of microglia. On the 69th day, the expression of iNOS and ARG1 both increased in the $25 \mathrm{mg} / \mathrm{kg}$ and $45 \mathrm{mg} / \mathrm{kg} P Q$ groups, and the expression of iNOS and ARG1 in the $45 \mathrm{mg} / \mathrm{kg} P Q$ group increased even more significantly. These findings, along with the results of $H E$ staining, suggest that $P Q$ could have long-term effects on microglia, and the neuronal damage in $\mathrm{SN}$ did not show complete recovery.

In sum, after acute PQ exposure, the poison quickly enters the brain through the blood-brain barrier, damaging neurons and stimulating the activation of microglia in the SN. We suspect that microglia may damage and repair neurons in $S N$ by changing phenotypic differentiation after acute $P Q$ exposure. The change in microglial phenotypic differentiation is related to the exposure dose and exposure time. Our results can thus explain the acute neurotoxicity of $P Q$ to some extent.

\section{Declarations}

\section{Data availability}

The datasets used and/or analysed during the current study are available from the corresponding author on reasonable request.

\section{Acknowledgments}


This study was supported by the Science and Technology Bureau of Hebei Province [grant numbers 132777140]. We thank Yujie Niu of the Public Health College of Hebei Medical University for their support and guidance. We thank Yansu Guo for help and advice.

\section{Funding}

All sources of funding for the research were declared. Bureau of Science and Technology of Hebei Province grant Song Bo (grant number312777140).

\section{Author contributions}

Wendi Zhang: Designed the study

Xiaobei Fan: Performed histological study

Zhuo Fan: Performed Wesern-blotting

Bailin Wu: Revised the study

Mengchao Wang and Wanyu Duan: Conducted, sampled and analyzed the study

Bo Song: Prepared, edited,and submitted the manuscript

Corresponding author

Correspondence to Bo Song

\section{Consent for publication}

All the authors have equally participated in this stydy and agreed to publish this work in this journal.

\section{Conflict of interest}

The authors declare that they have mo conflict of interest.

\section{Consent to publish}

Not applicable.

\section{Ethical approval}

This study was reviewed and approved by the Laboratory Animal Ethical and Welfare Committee Hebei Medical University.

\section{References}


Barlow, B.K., Thiruchelvam, M.J., Bennice, L., Cory-Slechta, D.A., Ballatori, N., et al, 2003. Increased synaptosomal dopamine content and brain concentration of paraquat produced by selective dithiocarbamates. Journal of Neurochemistry. 85, 1075-1086. https://doi.org/10.1046/j.14714159.2003.01773.x.

Bonneh-Barkay, D., Langston, W.J., Di Monte, D.A., 2005. Toxicity of redox cycling pesticides in primary mesencephalic cultures. Antioxid Redox Signal. 7, 649-653. https://doi.org/10.1089/ars.2005.7.649.

Brooks, A.I., Chadwick, C.A., Gelbard, H.A., Cory-Slechta, D.A., Federoff, H.J., 1999. Paraquat elicited neurobehavioral syndrome caused by dopaminergic neuron loss. Brain research. 823, 1-10. https://doi.org/10.1016/s0006-8993(98)01192-5.

Cassar, M., Issa, A.R., Riemensperger, T., Petitgas, C., Rival, T., et al, 2015. A dopamine receptor contributes to paraquat-induced neurotoxicity in Drosophila. Human molecular genetics. 24, 197-212. https://doi.org/10.1093/hmg/ddu430.

Chen, L., Na, R., Boldt, E., Ran, Q., 2015. NLRP3 inflammasome activation by mitochondrial reactive oxygen species plays a key role in long-term cognitive impairment induced by paraquat exposure. Neurobiology of aging. 36, 2533-2543. https://doi.org/10.1016/j.neurobiolaging.2015.05.018.

Cherry, J.D., Olschowka, J.A., O'Banion, M.K., 2014. Neuroinflammation and M2 phenotype: the good, the bad, and the inflamed. Journal of neuroinflammation. 11, 98. https://doi.org/10.1186/1742-2094-11-98.

Chhor, V., Le Charpentier, T., Lebon, S., Oré, M.V., Celador, I.L., et al, 2013. Characterization of phenotype markers and neuronotoxic potential of polarised primary microglia in vitro. Brain, behavior, and immunity. 32, 70-85. https://doi.org/10.1016/j.bbi.2013.02.005.

Crain, J.M., Nikodemova, M., Watters, J.J., 2013. Microglia express distinct M1 and M2 phenotypic markers in the postnatal and adult central nervous system in male and female mice. Journal of neuroscience research. 91, 1143-1151. https://doi.org/10.1002/jnr.23242.

Jha, M.K., Lee, W.H., Suk, K., 2016. Functional polarization of neuroglia: Implications in neuroinflammation and neurological disorders. Biochemical pharmacology. 103, 1-16. https://doi.org/10.1016/j.bcp.2015.11.003.

Kadowaki, T., Nakadate, K., Sakakibara, S., Hirata, K., Ueda, S., 2007. Expression of Iba1 protein in microglial cells of zitter mutant rat. Neuroscience letters. 411, 26-31.

https://doi.org/10.1016/j.neulet.2006.07.079.

Kim, S., Hwang, J., Lee, W.H., Hwang, D.Y., Suk, K., 2008. Role of protein kinase Cdelta in paraquat-induced glial cell death. Journal of neuroscience research. 86, 2062-2070. https://doi.org/10.1002/jnr.21643.

Lawson, L.J., Perry, V.H., Dri, P., Gordon, S., 1990. Heterogeneity in the distribution and morphology of microglia in the normal adult mouse brain. Neuroscience. 39, 151-170. https://doi.org/10.1016/0306- 
4522(90)90229-w.

Mantovani, A., Sica, A., Sozzani, S., Allavena, P., Vecchi, A., Locati, M., 2004. The chemokine system in diverse forms of macrophage activation and polarization. Trends in immunology. 25, 677-686. https://doi.org/10.1016/j.it.2004.09.015.

McCormack, A.L., Thiruchelvam, M., Manning-Bog, A.B., Thiffault, C., Langston, J.W., et al, 2002. Environmental Risk Factors and Parkinson's Disease: Selective Degeneration of Nigral Dopaminergic Neurons Caused by the Herbicide Paraquat. Neurobiology of Disease. 10,119-127. https://doi.org/10.1006/nbdi.2002.0507.

Mouton, P.R., Long, J.M., Lei, D..L, Howard ,V., Jucker, M., et al, 2002. Age and gender effects on microglia and astrocyte numbers in brains of mice. Brain research. 956, 30-35. https://doi.org/10.1016/s00068993(02)03475-3.

Nakagawa, Y., Chiba, K., 2014. Role of microglial m1/M2 phenotype in relapse and remission of psychiatric disorders and diseases. Pharmaceuticals (Basel, Switzerland). 7, 1028-1048. https://doi.org/10.3390/ph7121028.

Schindelin, J., Arganda-Carreras, I., Frise, E., Kaynig, V., Longair, M., et al, 2012. Fiji: an open-source platform for biological-image analysis. Nature methods. 9, 676-682. https://doi.org/10.1038/nmeth.2019.

Shimizu, K., Ohtaki, K., Matsubara, K., Aoyama, K., Uezono, T., et al, 2001. Carrier-mediated processes in blood-brain barrier penetration and neural uptake of paraquat. Brain research. 906, 135-142. https://doi.org/10.1016/s0006-8993(01)02577-x.

Sun, L., Yan, P.B., Zhang, Y.,Wei, L. Q., Li, G.Q., 2018. Effect of activated charcoal hemoperfusion on renal function in patients with paraquat poisoning. Experimental and therapeutic medicine. 15, 2688-2692. https://doi.org/10.3892/etm.2018.5712.

Wu, B., Song, B., Tian, S., Huo, S., Cui, C., et al, 2012. Central nervous system damage due to acute paraquat poisoning: a neuroimaging study with 3.0 T MRI. Neurotoxicology. 33, 1330-1337. https://doi.org/10.1016/j.neuro.2012.08.007.

Wu, B., Song, B., Yang, H., Huang, B., Chi, B., et al, 2013. Central nervous system damage due to acute paraquat poisoning: an experimental study with rat model. Neurotoxicology. 35, 62-70.

https://doi.org/10.1016/j.neuro.2012.12.001.

Wu, B.L., Song, B., 2013a. Reply to Dr. Jeffrey Brent. Neurotoxicology. 37, 220. https://doi.org/10.1016/j.neuro.2013.05.013.

Wu, B.L., Song, B., 2013b. Reply to drs. John andrew tomenson and clive campbell. Neurotoxicology. 36, 105. https://doi.org/10.1016/j.neuro.2013.02.011. 
Wu, X.F., Block, M.L., Zhang, W., Qin, L., Wilson, B., et al, 2005. The role of microglia in paraquat-induced dopaminergic neurotoxicity. Antioxid Redox Signal. 7, 654-661. https://doi.org/10.1089/ars.2005.7.654.

Young, K., Morrison, H., 2018. Quantifying Microglia Morphology from Photomicrographs of Immunohistochemistry Prepared Tissue Using ImageJ. Journal of visualized experiments : JoVE. 136,57648. https://doi.org/10.3791/57648.

Zhang, X.F., Thompson, M., Xu, Y.H., 2016. Multifactorial theory applied to the neurotoxicity of paraquat and paraquat-induced mechanisms of developing Parkinson's disease. Laboratory investigation; a journal of technical methods and pathology. 96, 496-507. https://doi.org/10.1038/labinvest.2015.161.

\section{Figures}

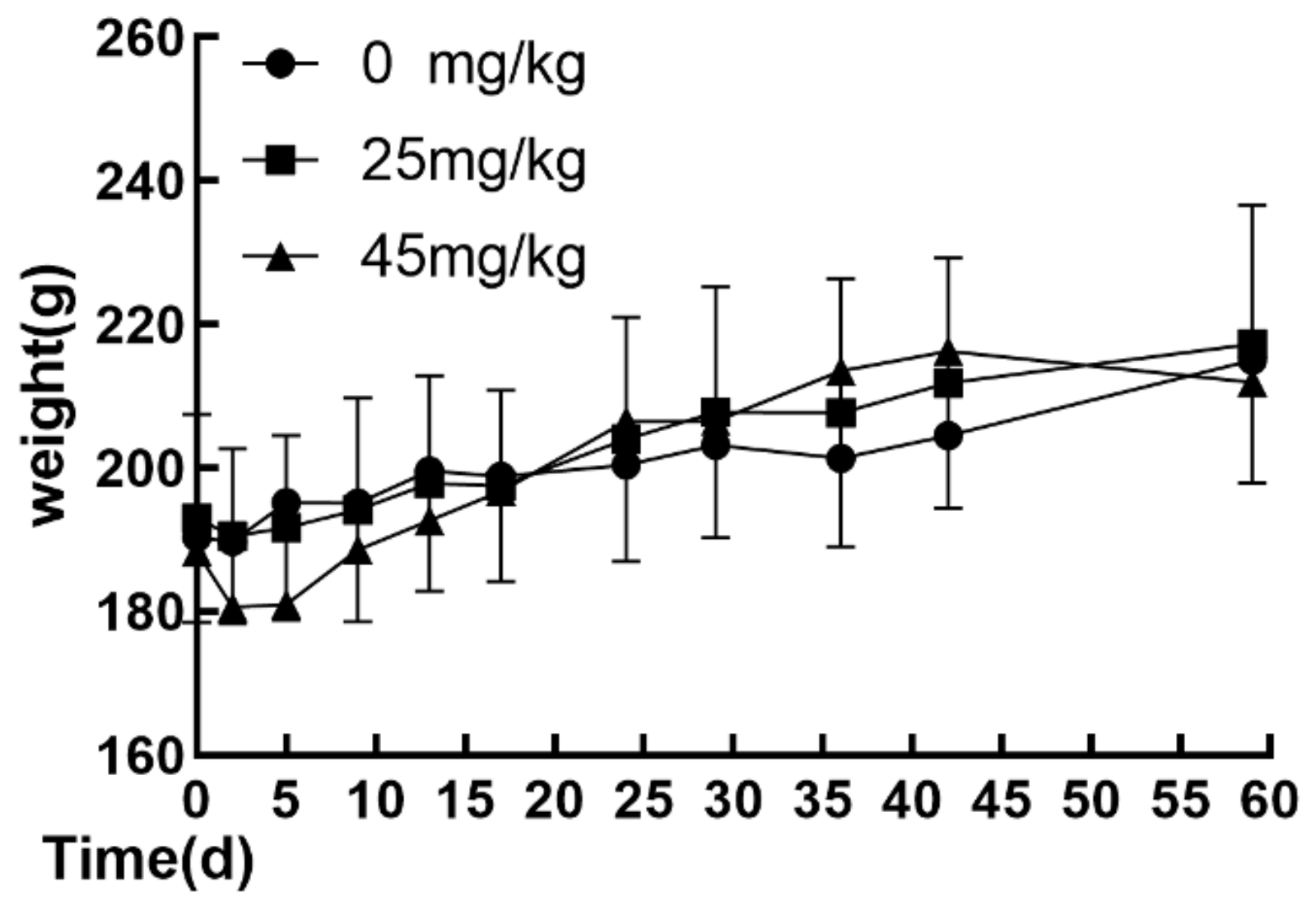

Figure 1

Changes in the body weight of rats after acute PQ exposure 


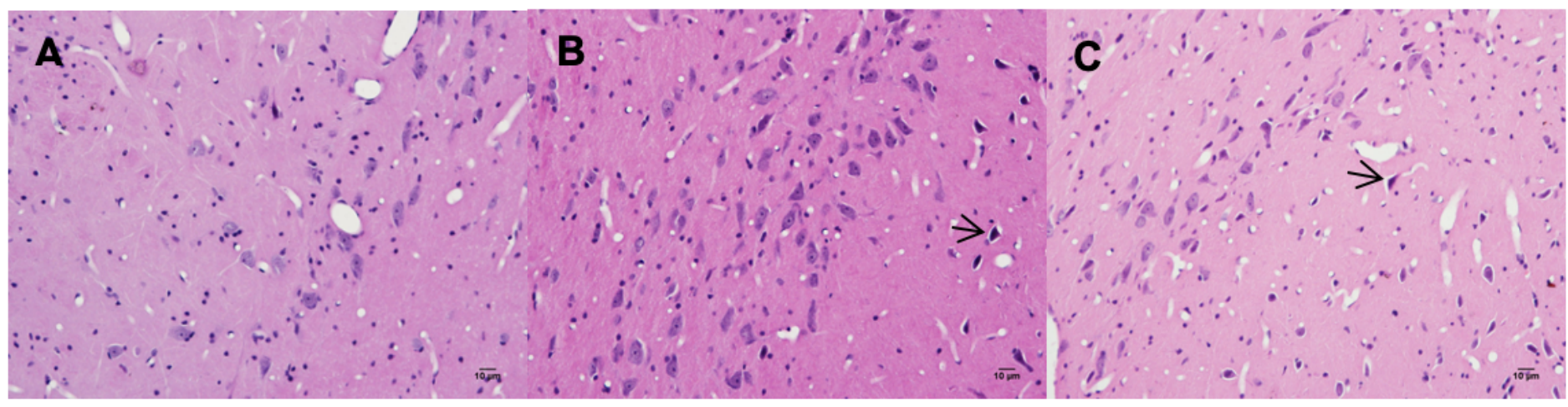

\section{Figure 2}

Histopathological changes in SN on the 18th day after acute PQ exposure (200x). (A) HE staining results of the control group; (B) HE staining results of the $25 \mathrm{mg} / \mathrm{kg}$ PQ exposure group; and (C) HE staining results of the $45 \mathrm{mg} / \mathrm{kg} P Q$ exposure group.

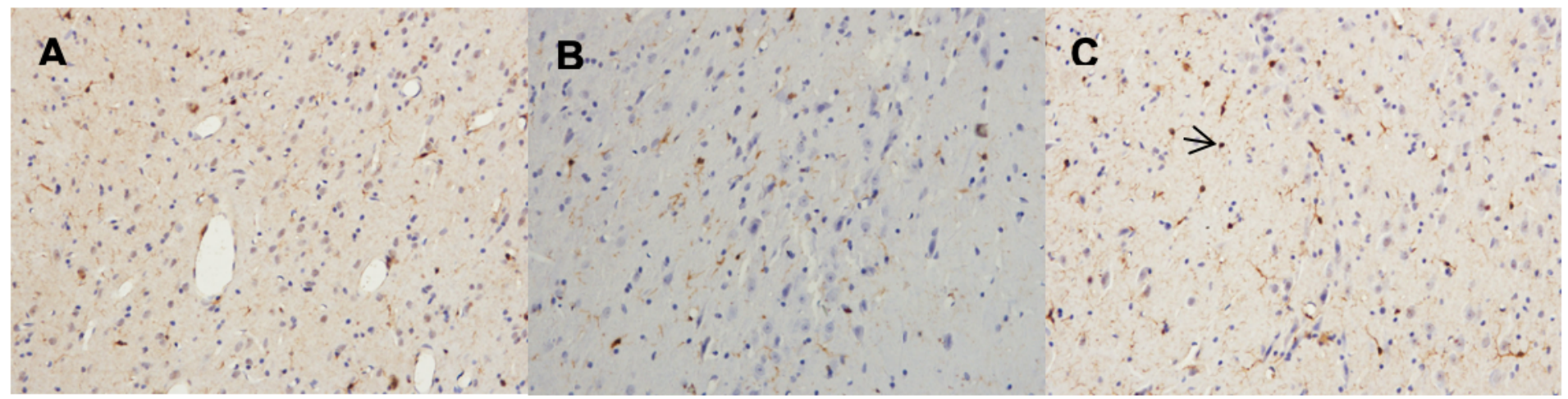

\section{Figure 3}

The morphology of Iba-1 positive cells on the 18th day after acute PQ exposure (200x). (A) IHC staining results of the control group; (B) IHC staining results of the $25 \mathrm{mg} / \mathrm{kg} P Q$ exposure group; and (C) IHC staining results of the $45 \mathrm{mg} / \mathrm{kg} P Q$ exposure group. 

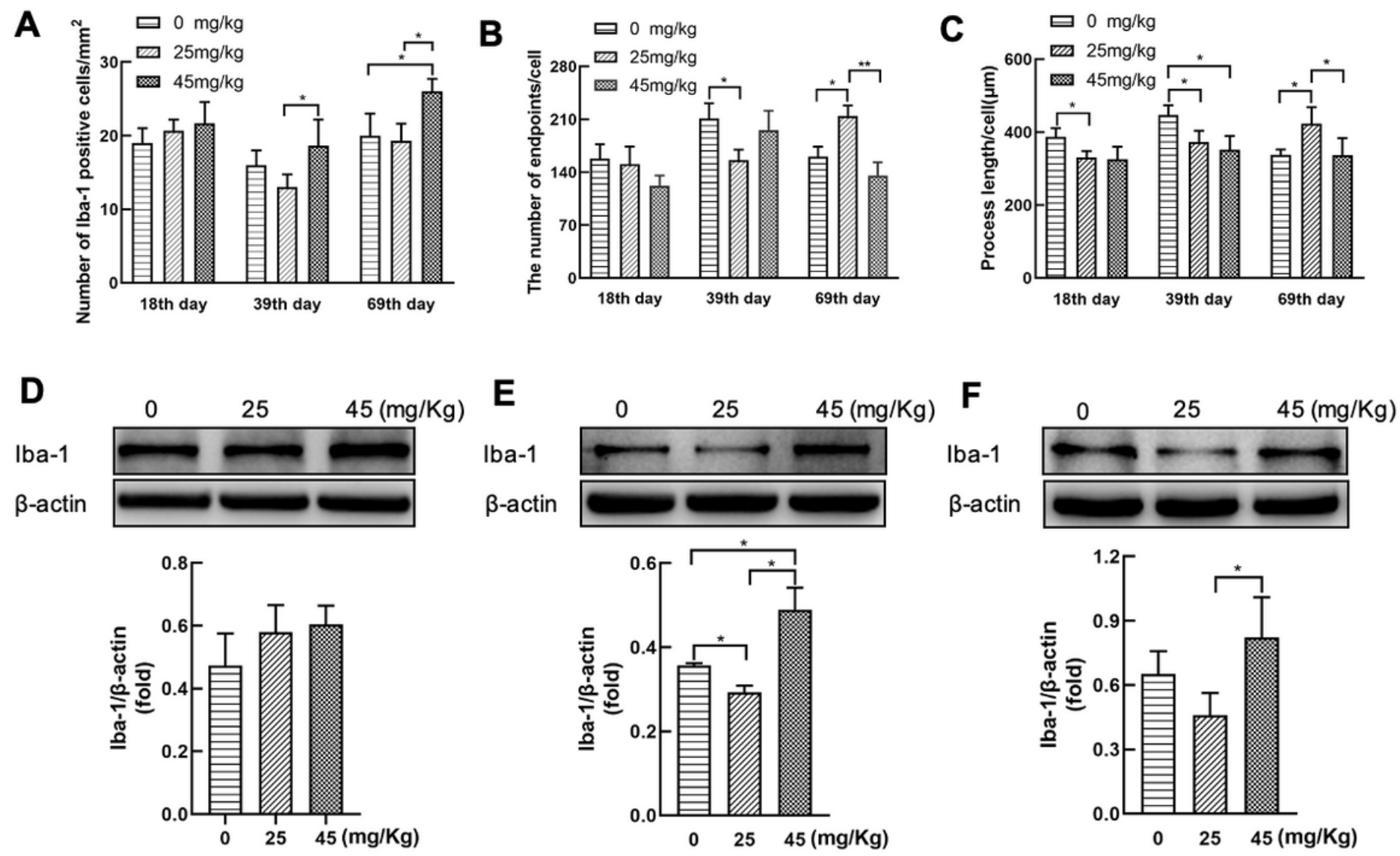

Figure 4

$P Q$ can induce changes in the number of activated microglia and microglia morphology. (A) The number of Iba-1 positive cells in the SN. (B) The average endpoints of Iba-1 positive cells in the SN. (C) The average process length of Iba-1 positive cells in the SN. (D-E) Relative changes in Iba-1 protein on the 18th, 39th and 69th day of exposure. $N=3,{ }^{*}<<0.05 .{ }^{*} \mathrm{P}<0.01$. Data are mean $\pm S D$. 


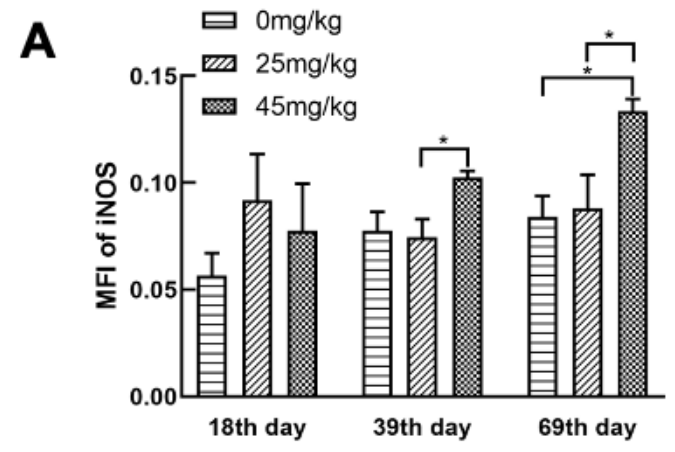

C
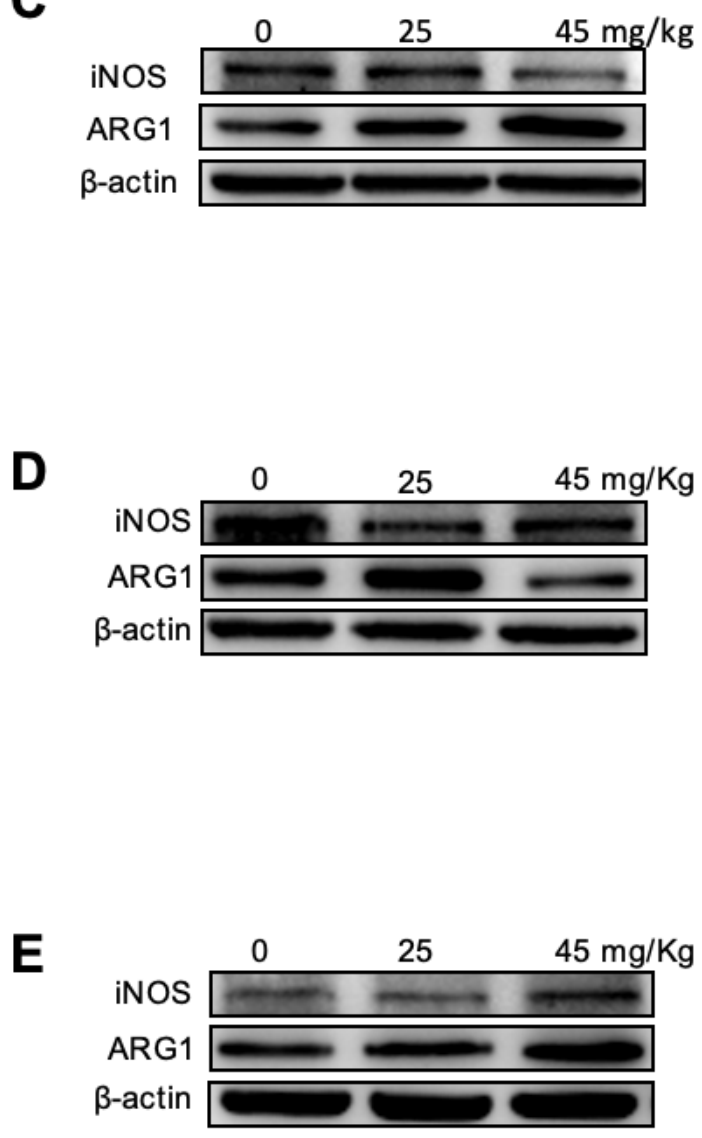
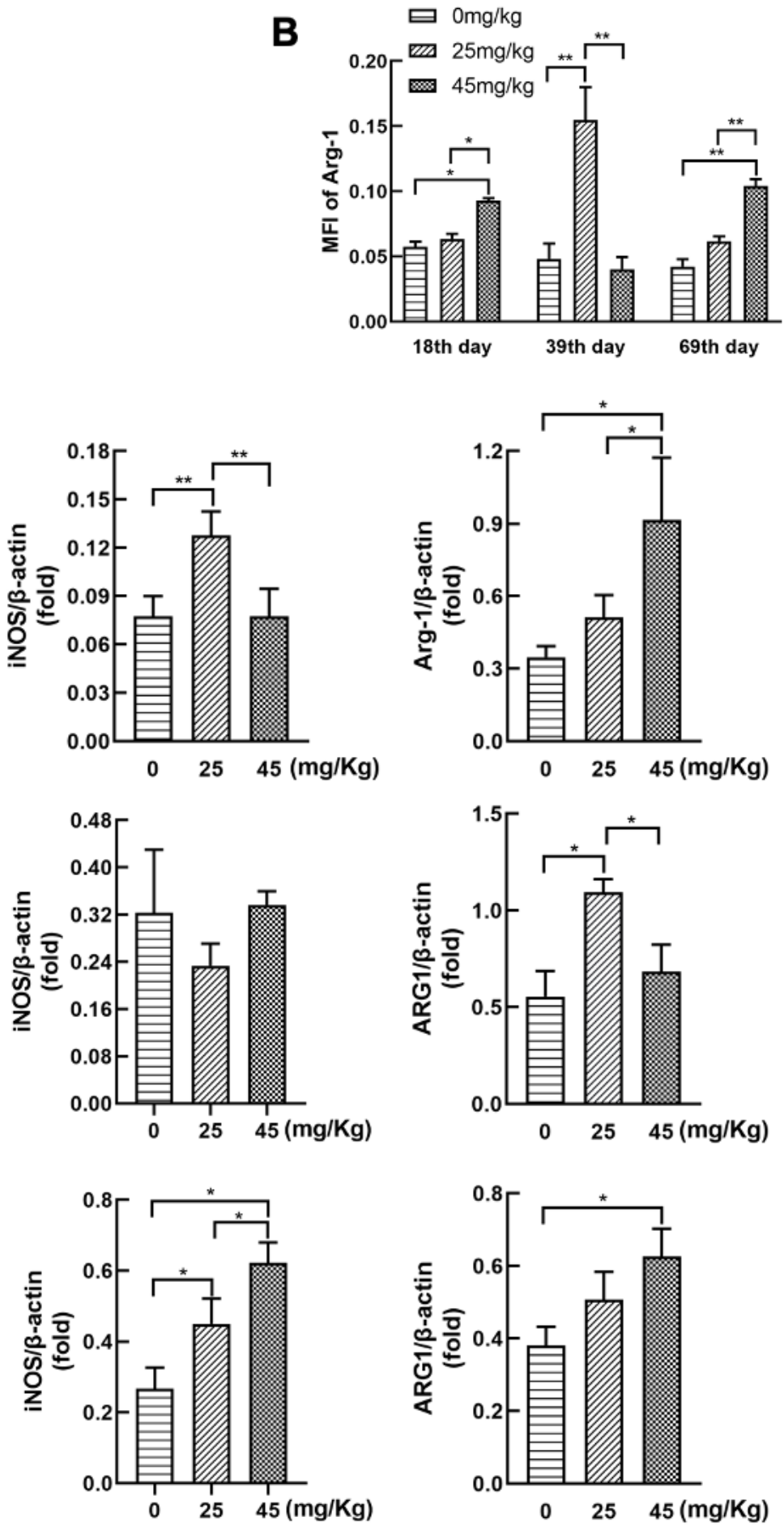

\section{Figure 5}

The expression of iNOS and ARG1 in the SN after acute PQ exposure. (A-B) The MFI of iNOS and ARG1 in the SN. (C-E) Relative protein expression of iNOS and ARG1 on the 18th, 39th and 69th day analyzed by western blot. $\mathrm{N}=3,{ }^{*} \mathrm{P}<0.05 .{ }^{*} \mathrm{P}<0.01$. Data are mean \pm SD. 